\title{
Prediction of Antagonistic Activity of $\beta$-Carboline and Its Derivatives Using Topological Descriptors
}

\author{
Anil Kumar Soni1 ${ }^{*}$, Gajendra Pal Singh², Vishnu Kumar Sahu ${ }^{3}$ \\ ${ }^{1}$ Department of Chemistry, Shia Post Graduate College, Lucknow, India \\ ${ }^{2}$ Department of Chemistry, Bareilly College, Bareilly, India \\ ${ }^{3}$ Department of Chemistry, Maharani Lal Kunwari Post Graduate College, Balrampur, India \\ Email: ^anilsony82@gmail.com, sgajendra888@gmail.com, vishnukr_sahu@rediffmail.com
}

How to cite this paper: Soni, A.K., Singh, G.P. and Sahu, V.K. (2021) Prediction of Antagonistic Activity of $\beta$-Carboline and Its Derivatives Using Topological Descriptors. Open Journal of Applied Sciences, 11, 577-584.

https://doi.org/10.4236/ojapps.2021.115041

Received: April 19, 2021

Accepted: May 25, 2021

Published: May 28, 2021

Copyright $\odot 2021$ by author(s) and Scientific Research Publishing Inc. This work is licensed under the Creative Commons Attribution International License (CC BY 4.0).

http://creativecommons.org/licenses/by/4.0/

\begin{abstract}
Prediction of antagonistic activity of $\beta$-carboline and its thirteen derivatives has been made using topological descriptors viz, connectivity index, and kappa shape index of different orders. For evaluation of values of descriptor, molecular modeling and geometry optimization of all the compounds were carried out with CAChe Pro software by opting semiempirical PM3 method using MOPAC 2002. For prediction of activity multiple linear regression analysis (MLR) was performed. MLR analysis has been made by Project Leader Software associated with CAChe by using the above descriptors as independent variables and biological activity as dependent variables. We were performed leave-one-out methods and the result reflected a direct relationship between biological activity and connectivity index of zero order, while indirect relationship with connectivity index of second order and thus connectivity index is a reliable descriptor to predict the biological activity of $\beta$-carboline and its various derivatives.
\end{abstract}

\section{Keywords}

$\beta$-Carboline, Antagonistic Activity, Connectivity Index and Kappa Shape Index, PM3

\section{Introduction}

In our previous publications, we have studied 1) PM3 based QSAR study of $\beta$ carbolines using electronegativity and absolute hardness as reactive parameters [1]; 2) stability index based quantitative structure-activity relationship study of $\beta$-carbolines [2];3) quantum chemical descriptor based study on $\beta$-carbolines 
binding to benzodiazepines receptor [3]; 4) correlation of binding affinity of $\beta$ carbolines on benzodiazepines receptor with UV- and IR-spectroscopic data [4]. But no study on $\beta$-carboline and its derivatives have been made using topological parameters. Hence, in this work, we have made a prediction of the binding affinities of $\beta$-carboline and its thirteen derivatives based on topological indices. Our research group has found reliable predicting ability of various topological indices on diverse sets of compounds of diverse biological activities [5]. The topological indices are molecular connectivity indices and shape index. Molecular connectivity is a method of molecular structure quantization in which weight counts of substructure fragments are incorporated into numerical indices such as size, branching, unsaturation, heteroatom content, and cyclicity, which are encoded [6]. Substructures for molecular skeleton are defined by the decomposition of the skeleton into fragments of atom (zero order, $m=0$ ) and one bond paths (first order, $m=1$ ). The calculation of indices begins with the reduction of the molecule to hydrogen-suppressed skeleton. Our study is based few selected topological descriptors, viz. 1) connectivity index of zero-order $\left.\left({ }^{0} \chi_{t}\right) ; 2\right)$ connectivity index of first order $\left.\left({ }^{1} \chi_{t}\right) ; 3\right)$ connectivity index of second order $\left.\left({ }^{2} \chi_{t}\right) ; 4\right)$ valence connectivity index of zero $\left.\left({ }^{0} \chi_{t}^{v}\right) ; 5\right)$ valence connectivity index of first or$\left.\operatorname{der}\left({ }^{1} \chi_{t}^{v}\right) ; 6\right)$ valence connectivity index of second order $\left({ }^{2} \chi_{t}^{v}\right)$; 7) kappa shape indix for first order $\left({ }^{1} K\right)$; 8) kappa shape indix for second order $\left({ }^{2} K\right)$; and 9) kappa shape indix for third order $\left({ }^{3} K\right)[7]$.

\section{Theory}

The evaluation of the above topological parameters is given below. The molecular connectivity indices are symbolized by ${ }^{m} \chi_{t}$ [7]. The connectivity index is given by Equation (1):

$$
{ }^{m} \chi_{t}=\sum_{i=1}^{N s}{ }^{m} C_{i}
$$

and ${ }^{m} C_{i}$ is given by Equation (2):

$$
{ }^{m} C_{i}=\prod_{k=1}^{m+1}\left(\delta_{k}\right)^{-0.5}
$$

where $m=1$ for first order, $m=2$ for second order and $m=3$ for third order. Similarly the valence connectivity index is given by Equation (3):

$$
{ }^{m} \chi_{t}^{V}=\sum_{i=1}^{N s}{ }^{m} C_{i}^{V}
$$

and ${ }^{m} C_{i}^{V}$ is given by Equation (4):

$$
{ }^{m} C_{i}^{V}=\prod_{k=1}^{m+1}\left(\delta_{k}^{V}\right)^{-0.5}
$$

where $m=1$ for first order, $m=2$ for second order and $m=3$ for third order.

Kappa shape indices are also a method of molecular structure quantization in which attributes of molecular shape are encoded into kappa values ( ${ }^{1} K$ for first order, ${ }^{2} K$ for second order, ${ }^{3} K$ for third order) [5]. The first, second and third order kappa shape indices are given by Equation (5)-(8): 


$$
\begin{gathered}
{ }^{1} K=\frac{A(A-1)^{2}}{\left({ }^{1} P_{i}\right)^{2}} \\
{ }^{2} K=\frac{(A-1)(A-2)^{2}}{\left({ }^{2} P_{i}\right)^{2}} \\
{ }^{3} K=\frac{(A-1)(A-3)^{2}}{\left({ }^{3} P_{i}\right)^{2}} \text { if } A \text { is odd } \\
{ }^{3} K=\frac{(A-3)(A-2)^{2}}{\left({ }^{3} P_{i}\right)^{2}} \text { if } A \text { is even }
\end{gathered}
$$

where $P_{i}$ is the length of paths of bond length " $i$ " in the hydrogen suppressed molecule and " $A$ " is the number of non-hydrogen atoms in the molecule.

\section{Materials and Methods}

The study materials of this work are $\beta$-carboline and its thirteen derivatives as listed in Table 1 . The biological activity of these compounds were measured by three different methods viz., IC50 inhibition of $[3 \mathrm{H}]$ diazepam, $\mathrm{IC}_{50}$ antagonistic activity, and $\mathrm{IC}_{50}$ binding affinities to displace $50 \%$ of [3H]flunitrazepam on benzodiazepines receptor [8]. But in this work, we have selected $\mathrm{IC}_{50}$ antagonistic activity for the study. For prediction of antagonistic activity molecular modeling and geometry optimization [9] of all the compounds were carried out with CAChe Pro software by opting semiempirical PM3 method using MOPAC 2002 [10]. The MLR analysis has been made by Project Leader Software associated with CAChe by using the above descriptors. The reliability of the proposed model was judged by calculating the values of the correlation coefficient $\left(r^{2}\right)$ and cross validation coefficient $\left(r_{C V}^{2}\right)[11]$.

\section{Results and Discussion}

Benzodiazepines (BDZs) are the drugs of choice in the pharmacotherapy of anxiety and related emotional disorders, sleep disorders, status epilepticus, and other convulsive states; they are used as centrally acting muscle relaxants, for premedication, and as inducing agents in anesthesiology [8]. They act via the benzodiazepine receptor site (BzR) on the $\gamma$-aminobutyric acid receptor $\left(\mathrm{GABA}_{\mathrm{A}}\right)$ family [8]. There are relatively several structural classes of Non-Benzodiazepine (Non-BDZ) compounds that have a reasonable affinity for the BZR and show pharmacologic activity in vivo [8]. But, in this work, we have made QSAR study of a series of $\beta$-carboline derivatives as Non-BDZ molecules whose biological activities are reported in terms of $\mathrm{IC}_{50}$ antagonistic activity binding to the benzodiazepine receptor. $\beta$-Carbolines posssess a broad spectrum of pharmacological actions (as muscle relaxants) mediated via occupation of BzR in the central nervous system [8]. Survey of the literature shows that biological activity of several $\beta$-carboline derivatives has been measured and discussed in detail [8]. The survey of the literatures also indicates that no prediction of activity of 
$\beta$-carboline derivatives has been made with the help of above selected topological descriptors. The trends of topological descriptors with antagonistic activity are graphically depicted in Figure 1.

Table $1 . \mathrm{IC}_{50}$ antagonistic activity of $\beta$-Carboline and its thirteen derivative binding to benzodiazepines receptor [8].

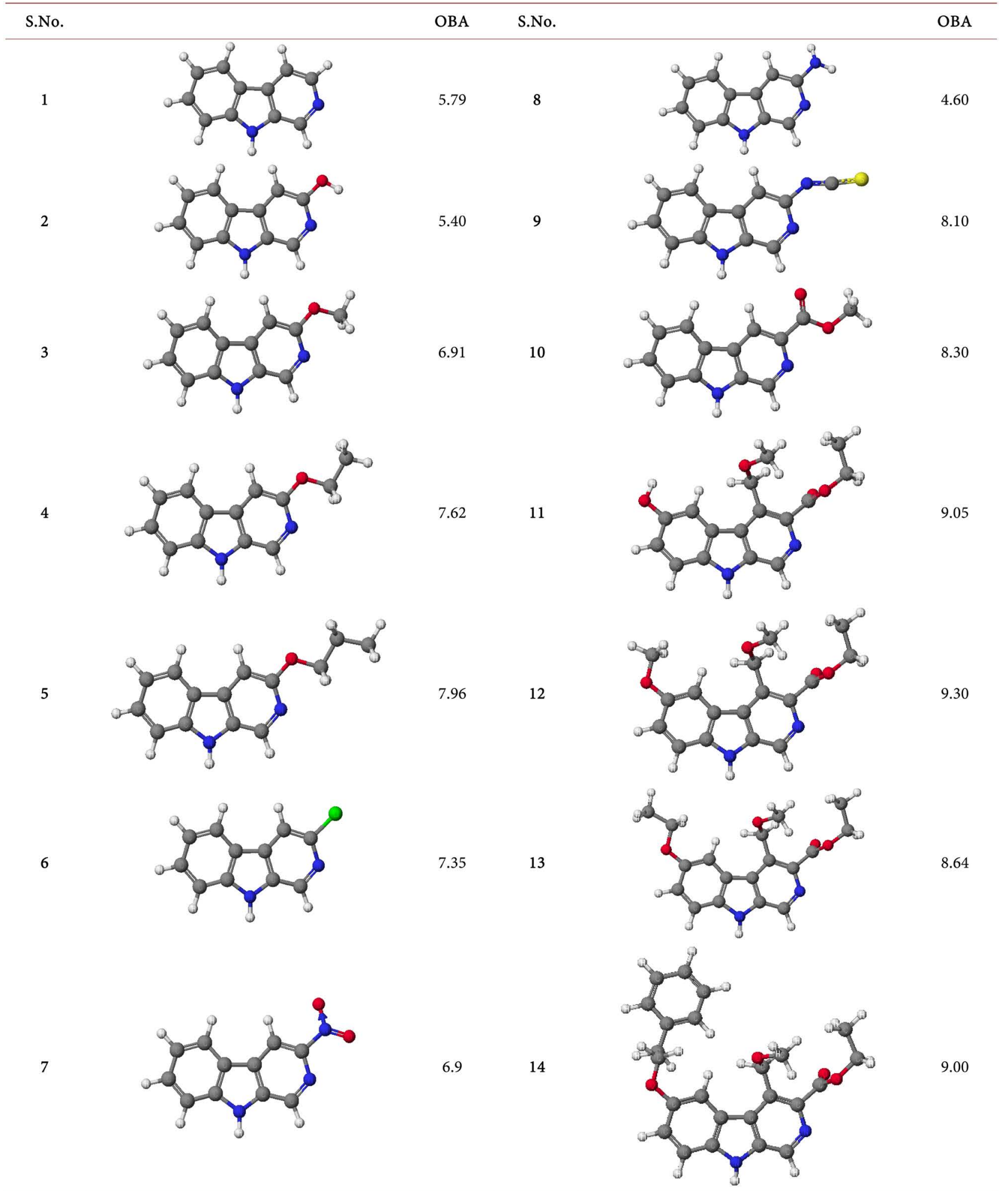



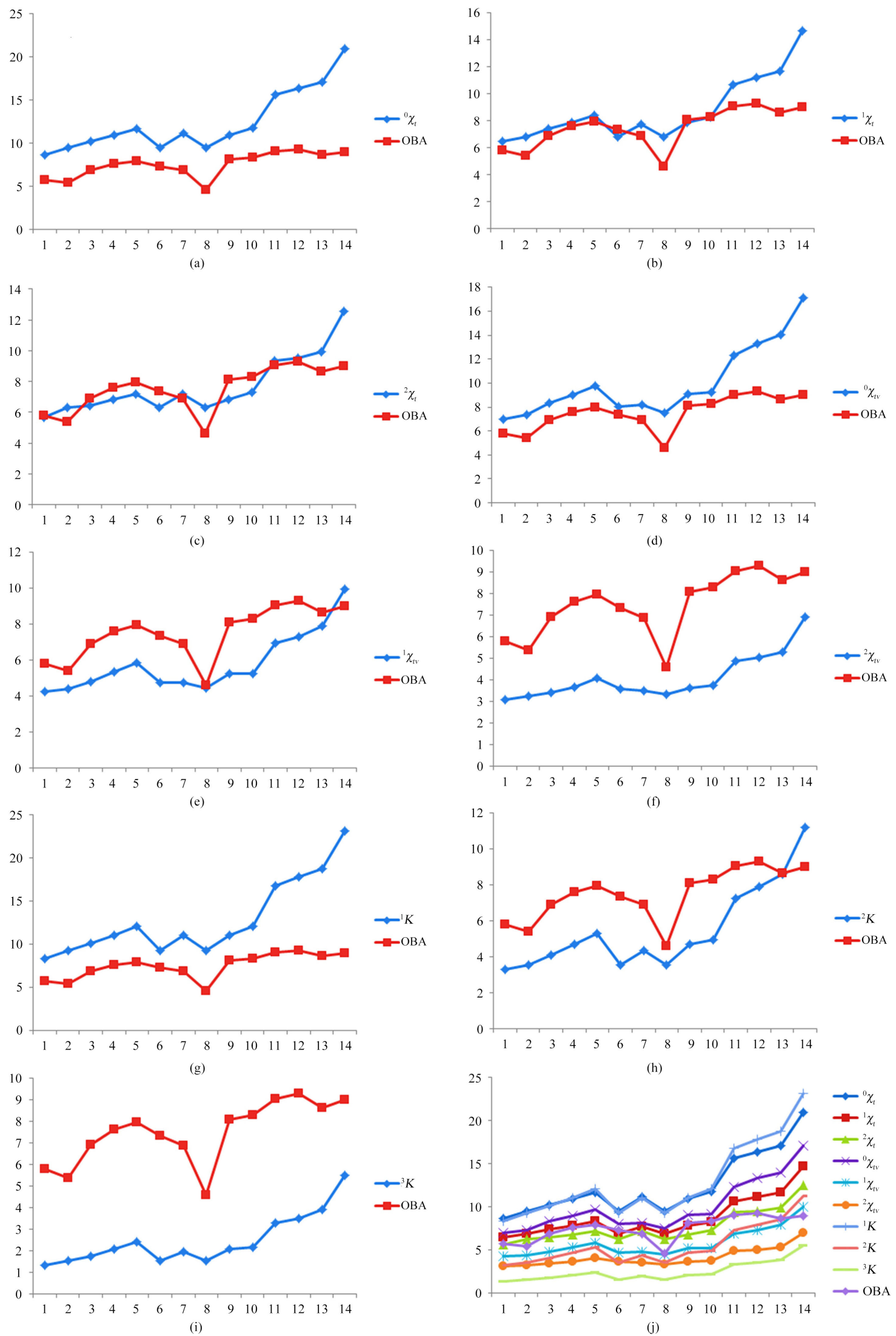

Figure 1. Trends of descriptors with biological activity. 
For prediction of antagonistic activity multiple regression analysis has been performed using topological descriptors used as independent variables and biological activity as dependent variable. Through leave-out-one method a number of MLR equations have been developed using topological descriptors not more than three. when $k=1$ then the reliable modal is:

$$
\begin{gathered}
\mathrm{PBA}=0.256 \times{ }^{0} \chi_{t}^{v}+4.225 \\
r_{C V}^{2}=0.308 \\
r^{2}=0.571
\end{gathered}
$$

when $k=2$ then the reliable model obtained by different combination of descriptors is:

$$
\begin{gathered}
\mathrm{PBA}=2.389 \times{ }^{0} \chi_{t}-3.847 \times{ }^{2} \chi_{t}+7.486 \\
r_{C V}^{2}=0.648 \\
r^{2}=0.736
\end{gathered}
$$

when $k=3$ then the reliable model obtained by different combination of descriptors is:

$$
\begin{gathered}
\mathrm{PBA}=1.869 \times{ }^{1} K-0.246 \times{ }^{2} K-3.724 \times{ }^{3} K+5.645 \\
r_{C V}^{2}=0.583 \\
r^{2}=0.736
\end{gathered}
$$

\begin{tabular}{|c|c|c|c|c|c|c|c|c|c|c|c|}
\hline \multirow{2}{*}{ S.No. } & \multicolumn{9}{|c|}{ Descriptors } & \multirow{2}{*}{ OBA } & \multirow{2}{*}{ PA } \\
\hline & ${ }^{0} \chi_{t}$ & ${ }^{1} \chi_{t}$ & ${ }^{2} \chi_{t}$ & ${ }^{0} \chi_{t}^{v}$ & ${ }^{1} \chi_{t}^{v}$ & ${ }^{2} \chi_{t}^{v}$ & ${ }^{1} K$ & ${ }^{2} K$ & ${ }^{3} K$ & & \\
\hline 1 & 8.673 & 6.449 & 5.652 & 6.989 & 4.254 & 3.092 & 8.32 & 3.293 & 1.333 & 5.79 & 6.462 \\
\hline 2 & 9.544 & 6.843 & 6.286 & 7.359 & 4.399 & 3.259 & 9.242 & 3.539 & 1.547 & 5.40 & 6.103 \\
\hline 3 & 10.251 & 7.381 & 6.455 & 8.32 & 4.788 & 3.443 & 10.173 & 4.108 & 1.744 & 6.91 & 7.142 \\
\hline 4 & 10.958 & 7.881 & 6.836 & 9.027 & 5.375 & 3.672 & 11.111 & 4.704 & 2.08 & 7.62 & 7.368 \\
\hline 5 & 11.665 & 8.381 & 7.189 & 9.734 & 5.875 & 4.087 & 12.055 & 5.325 & 2.42 & 7.96 & 7.697 \\
\hline 6 & 9.544 & 6.843 & 6.286 & 8.045 & 4.742 & 3.61 & 9.242 & 3.539 & 1.547 & 7.35 & 6.103 \\
\hline 7 & 11.121 & 7.754 & 7.185 & 8.175 & 4.764 & 3.516 & 11.111 & 4.349 & 1.966 & 6.90 & 6.413 \\
\hline 8 & 9.544 & 6.843 & 6.286 & 7.489 & 4.464 & 3.325 & 9.242 & 3.539 & 1.547 & 4.60 & 6.103 \\
\hline 9 & 10.958 & 7.881 & 6.836 & 9.083 & 5.235 & 3.644 & 11.111 & 4.704 & 2.08 & 8.10 & 7.368 \\
\hline 10 & 11.828 & 8.292 & 7.327 & 9.228 & 5.242 & 3.777 & 12.055 & 4.938 & 2.172 & 8.30 & 7.558 \\
\hline 11 & 15.69 & 10.651 & 9.375 & 12.343 & 6.937 & 4.882 & 16.844 & 7.266 & 3.299 & 9.05 & 8.905 \\
\hline 12 & 16.397 & 11.189 & 9.544 & 13.304 & 7.325 & 5.064 & 17.811 & 7.92 & 3.52 & 9.30 & 9.944 \\
\hline${ }^{\mathrm{a}} 13$ & 17.104 & 11.689 & 9.925 & 14.011 & 7.913 & 5.293 & 18.781 & 8.59 & 3.908 & 8.64 & 10.169 \\
\hline 14 & 20.924 & 14.707 & 12.571 & 17.105 & 9.97 & 6.944 & 23.168 & 11.228 & 5.507 & 9.00 & 9.116 \\
\hline
\end{tabular}

Among the above MLR equations Equation (10) is best model as clear from the correlation coefficient $\left(r^{2}\right)$ and cross validation coefficient $\left(r_{C V}^{2}\right)$ values. Predicted activity as obtained from this model is also tabulated in Table 2, while the trends of observed and predicted activity are graphically presented in Figure 2.

Table 2. Values of topological descriptors, observed biological activity and predicted biological activity.

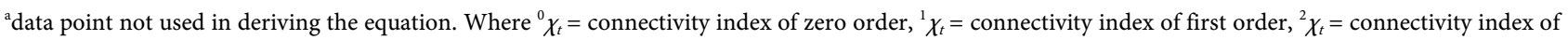
second order, ${ }^{0} \chi_{t}^{v}=$ valence connectivity index of zero, ${ }^{1} \chi_{t}^{v}=$ valence connectivity index of first order, ${ }^{2} \chi_{t}^{v}=$ valence connectivity index of second order, ${ }^{1} K=$ kappa shape indix for first order, ${ }^{2} K=$ kappa shape indix for second order, ${ }^{3} K=$ kappa shape indix for third order, OBA $=$ observed biological activity in term of $\mathrm{IC}_{50}$ antagonistic activity and $\mathrm{PBA}=$ predicted biological activity. 


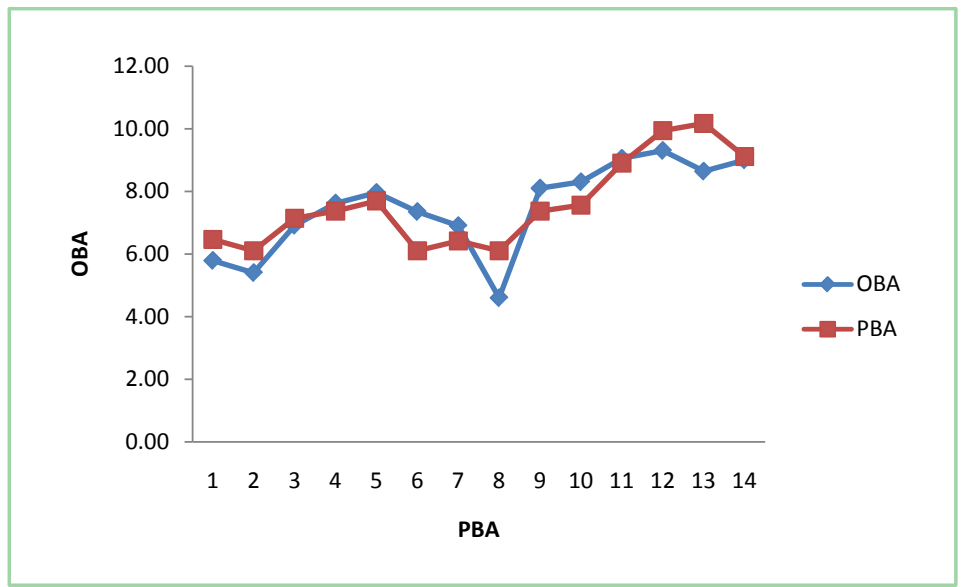

Figure 2. Trends of OBA and PBA.

\section{Conclusion}

The study concluded that the connectivity indexes of zero-order and connectivity second order are reliable descriptors to predict the biological activity of $\beta$ carboline and its various derivatives. The study also reflected a direct relationship between biological activity and connectivity index of zero-order (positive value of descriptor coefficient), while the indirect relationship with connectivity index of second order (negative value of descriptor coefficient).

\section{Acknowledgements}

The authors are thankful to Principal, Maharani Lal Kunwari Post Graduate College, Balrampur for laboratory facilities and to respected Dr. P. P. Singh for valuable discussion and suggestions.

\section{Conflicts of Interest}

The authors declare no conflicts of interest regarding the publication of this paper.

\section{References}

[1] Singh, P.P, Soni, A.K. and Sahu, V.K. (2009) PM3 Based QSAR Study of $\beta$-Carbolines Using Electronegativity and Absolute Hardness as Reactive Parameters. Organic Chemistry-An Indian Journal, 5, 55-58.

[2] Sahu, V.K., Soni, A.K. and Singh, P.P. (2010) Stability Index Based Quantitative Structure-Activity Relationship Study of $\beta$-Carbolines. Asian Journal Chemistry, 22, 3025-3035.

[3] Soni, A.K., Sahu, V.K. and Singh, P.P. (2011) Quantum Chemical Descriptor Based Quantitative Structure-Activity Relationship Study of $\beta$-Carbolines. Asian JournalChemistry, 23, 671-675.

[4] Soni, A.K., Sahu, S., Sahu, V.K. and Singh, P. (2015) Correlation of Binding Affinity $\beta$-Carbolines on Benzodiazepines Receptor with UV- and IR-Spectroscopic Data. International Journal of Advanced Research, 3, 1336-1343.

[5] Singh, P.P., Sahu, V.K., Singh, R.K. and Singh, P. (2008) Hydrophobic, Topological 
and Steric Parameter Based QSAR Study on Peptidic HIV-Protease Inhibitors. Organic Chemistry-An Indian Journal, 4, 284-294.

[6] Randi, M. (1975) Characterization of Molecular Branching. Journal of American Chemical Society, 97, 6609-6615. https://doi.org/10.1021/ja00856a001

[7] Kier, L.B. and Hall, L.H. (1986) Molecular Connectivity in Structure-Activity Analysis. John Wiley \& Sons, New York.

[8] Gupta, S.P. and Paleti, A. (1996) Quantitative Structure-Activity Relationship Studies on Benzodiazepine Receptor Binding of Some Nonbenzodiazepine Series of Ligands. Quantitative Structure-Activity Relationships, 15, 12-16. https://doi.org/10.1002/qsar.19960150104

[9] Stewart, J.J.P. (1989) Optimization of Parameters for Semiempirical Methods I. Method. Journal of Computational Chemistry, 10, 209-220.

[10] Stewart, J.J.P. (1990) MOPAC: A General Molecular Orbital Program. Journal of Computer-Aided Molecular Design, 4, 1-103. https://doi.org/10.1007/BF00128336

[11] Myers, R.H. (1990) Classical and Modern Regression Application. 2nd Edition, Duxbury Press, California, USA. 